\section{Schutz und Revitalisierung von Fließgewässern}

Das Verbesserungsgebot der EU-Wasserrahmenrichtlinie führte - in Zusammenschau mit anderen naturschutzrelevanten Richtlinien - in zahlreichen europäischen Ländern zu verstärkten Sanierungsbzw. Revitalisierungsmaßnahmen an Fließgewässern. Ebenso wurde in den vergangenen Jahren dem Schutz ökologisch wertvoller Flüsse hohe Bedeutung im Gewässermanagement eingeräumt, was sich in den Aktivitäten zur Entwicklung von übergeordneten Schutzstrategien und Planungsvorgaben sowohl in Österreich als auch europaweit zeigt.

Die vorliegende Ausgabe der „Österreichischen Wasser- und Abfallwirtschaft“ widmet sich diesen Themen mit fünf Beiträgen, die wesentliche Erkenntnisse aus Fallstudien zu Fragen von Schutz und Revitalisierung von Fließgewässern vorstellen und damit auch einen Beitrag zur Diskussion aktueller methodischer Herangehensweisen liefern.

In dem Beitrag von Scheikl et al. werden Konzepte und Fallbeispiele zum Schutz ökologisch sensibler Fließgewässer dokumentiert. Die Autorlnnen stellen methodische Herangehensweisen zur Priorisierung von Schutzmaßnahmen auf nationaler (Österreich) als auch regionaler Ebene (Einzugsgebiet Tiroler Inn) dar. Ein Fallspiel ist dabei der Analyse aller österreichischen Fließgewässer mit einem Einzugsgebiet $>500 \mathrm{~km}^{2}$ zur Kategorisierung unterschiedlicher ökologischer Wertigkeiten gewidmet. In den beiden anderen Beispielen werden energiewirtschaftliche und ökologisch-naturschutzfachliche Ansprüche mittels einer Vielzahl an fachspezifischen Kriterien einander gegenübergestellt und evaluiert - einmal unmittelbar bezogen auf österreichweit geplante Wasserkraftnutzungen (102 Projekte), ein weiteres Mal in Form eines einzugsgebietsbezogenen Planungsansatzes zur Schutzpriorisierung sensibler Gewässerstrecken des Tiroler Inn-Systems. Da in Österreich der weitere Ausbau der Wasserkraft ein bedeutendes Thema ist, nicht zuletzt auch aufgrund der Vorgaben der EU-ErneuerbareEnergien-Richtlinie, gilt es, in Form strategischer, vorausschauender Planungen eine transparente Abwägung unterschiedlicher Interessen zu gewährleisten.

Maßnahmen zur Wieder-Vernetzung von Fluss- und Auenlebensräumen wurden in jüngster Zeit vermehrt realisiert - mit dem übergeordneten Ziel, der Entkoppelung von Fluss und Auen entgegenzuwirken. Was oftmals fehlt - neben detaillierten Grundlagendaten über Formen und Funktionen der FlussUmland-Interaktionen (siehe auch Beitrag Hohensinner \& Jungwirth in diesem Heft) - sind Monitoring-Ergebnisse solcher Managementmaßnahmen ebenso wie Prognose-Modelle, um die zukünftigen Folgewirkungen abschätzen zu können. Am Beispiel der Unteren Lobau/Donau wurden von Trauner et al. (,Integrierte gewässerökologische Modellansätze zur Beurteilung von Gewässervernetzungsvarianten“) drei Management-Szenarien mittels der Modellierung der Habitatpräferenzen ausgewählter Tier- und Pflanzenarten analysiert. Anhand der Modellierungsergebnisse konnte der Einfluss unterschiedlicher hydrologischer Vernetzungsmaßnahmen auf die Biozönosen beschrieben werden. Die Studienergebnisse liefern damit eine wertvolle Grundlage für die Maßnahmenplanung und Entscheidungsfindung im Gewässermanagement.

Melcher at al. untersuchten im Rahmen eines Forschungsprojektes des österreichischen Klima- und Energiefonds den „Einfluss der Ufervegetation auf die Wassertemperatur unter gewässertypspezifischer Berücksichtigung von Fischen und benthischen Evertebraten“. Die Autoren konnten anhand der Fallbeispiele Lafnitz und Pinka aufzeigen, dass bereits der Anstieg der Wassertemperatur um $2{ }^{\circ} \mathrm{C}$ zu einer Verschiebung der Artenzusammensetzung führte. Gleichzeitig wurde belegt, dass die Ufervegetation in Hinblick auf die zukünftig zu erwartenden Auswirkungen des Klimawandels auf das thermische Regime im Zusammenwirken mit naturnahen aquatischen Habitaten eine besondere Bedeutung besitzt.

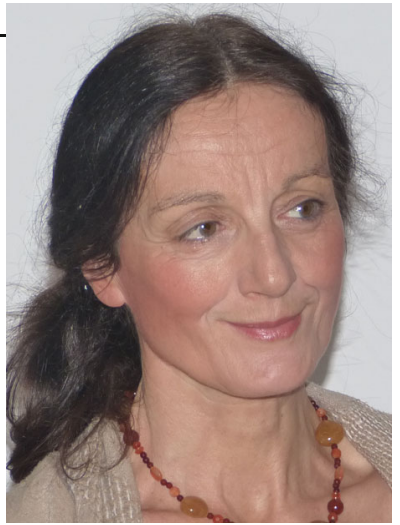

ao.Univ.-Prof. DI Dr. Susanne Muhar 
Der Beitrag von Hohensinner \& Jungwirth widmet sich der „Unbekannten dritten Dimension: Geländehöhen, Gewässertiefen und Dynamik österreichischer Donaulandschaften vor der Regulierung“. Die Ergebnisse geben Aufschluss über verschiedene, ökomorphologisch relevante Geländezonen innerhalb des Augebietes, deren naturräumliche Charakteristika und ökologische Funktionen. Die ursprünglich hohe Umlagerungsintensität, durch die große Anteile der aquatischen und terrestrischen Habitate in vergleichsweise kurzen Zeiträumen regeneriert und verjüngt wurden, ist Voraussetzung zur Aufrechterhaltung eines heterogenen Habitatgefüges. Die Daten dienen als wesentliche Grundlage für die Definition von Referenzbedingungen zur Implementierung und Evaluierung typspezifischer, hydromorphologischer Sanierungsmaßnahmen.

Wie sehr es gelingt, das Systemverständnis über Prozesse in Flusslandschaften bei Jugendlichen zu fördern, war zentraler Forschungsgegenstand des Sparkling-Science-Projektes „Was? Wie? Warum? Jugendliche erforschen Flusslandschaften“. Poppe und ihre Co-Autorlnnen setzten im Fallbeispiel Traisen unterschiedliche Methoden ein, durch die sich die SchülerInnen Wissen über die Komplexität von Fluss-Auen-Systemen aneignen konnten. Die Auswertung von Wissenstests sowie der Modellierungsergebnisse der SchülerInnen vor und nach den gemeinsamen Aktivitäten belegen ein maßgeblich gesteigertes Verständnis für wichtige kausale Zusammenhänge in der Flusslandschaft Traisen. Zudem konnte gezeigt werden, dass das Konzept der Ökosystemleistungen in diesem Fallbeispiel eine geeignete Methode zur Wissensvermittlung von komplexen Zusammenhängen in Flusslandschaften darstellt.

ao.Univ.-Prof. DI Dr. S. Muhar ( $₫)$

Department Wasser-Atmosphäre-

Umwelt, Institut für Hydrobiologie und

Gewässermanagement, Universität

für Bodenkultur Wien, Max-Emanuel-

Straße 17,

1180 Wien, Österreich

E-Mail: susanne.muhar@boku.ac.at 